\title{
ON THE LOCATING-CHROMATIC NUMBERS OF SUBDIVISIONS OF FRIENDSHIP GRAPH
}

\author{
Brilly Maxel Salindeho ${ }^{1}$, Hilda Assiyatun $^{2}$, Edy Tri Baskoro $^{3}$ \\ ${ }^{1}$ Department of Mathematics, Mulawarman University, \\ brillyms@gmail.com \\ ${ }^{2,3}$ Department of Mathematics, Bandung Institute of Technology
}

\begin{abstract}
Let $c$ be a $k$-coloring of a connected graph $G$ and let $\pi=$ $\left\{C_{1}, C_{2}, \ldots, C_{k}\right\}$ be the partition of $V(G)$ induced by $c$. For every vertex $v$ of $G$, let $c_{\pi}(v)$ be the coordinate of $v$ relative to $\pi$, that is $c_{\pi}(v)=$ $\left(d\left(v, C_{1}\right), d\left(v, C_{2}\right), \ldots, d\left(v, C_{k}\right)\right)$, where $d\left(v, C_{i}\right)=\min \left\{d(v, x) \mid x \in C_{i}\right\}$. If every two vertices of $G$ have different coordinates relative to $\pi$, then $c$ is said to be a locating $k$-coloring of $G$. The locating-chromatic number of $G$, denoted by $\chi_{L}(G)$, is the least $k$ such that there exists a locating $k$-coloring of $G$. In this paper, we determine the locating-chromatic numbers of some subdivisions of the friendship graph $F r_{t}$, that is the graph obtained by joining $t$ copies of 3 -cycle with a common vertex, and we give lower bounds to the locating-chromatic numbers of few other subdivisions of $F r_{t}$.
\end{abstract}

Keywords: friendship graph, locating-chromatic number, locating coloring, subdivision

\section{INTRODUCTION}

The concept of locating-chromatic number was first studied by Chartrand et al. [1] by combining the concept of graph partition dimension and graph coloring. The locating-chromatic numbers of some classes of graphs were studied, especially recently for certain Barbell graphs in [2], Halin graphs in [3], and graphs resulting from certain operations of other graphs, such as join of graphs in [4] and Cartesian product of graphs in [5]. Trees with certain locating-chromatic number were also studied in [6] and [7]. Bounds for locating-chromatic numbers of trees and subdivisions of graph on one edge were also established in [8] and [9], respectively.

Suppose that $G=(V, E)$ is a simple connected graph. Let $c$ be a $k$-coloring on $G$ and let $\pi=\left\{C_{1}, C_{2}, \ldots, C_{k}\right\}$ be the partition of $V=V(G)$ induced by $c$. For every vertex $v$ of $G$, let $c_{\pi}(v)=\left(d\left(v, C_{1}\right), d\left(v, C_{2}\right), \ldots, d\left(v, C_{k}\right)\right)$ be the coordinate

2020 Mathematics Subject Classification: 05C15, 05C70.

Received: 01-11-2018, accepted: 01-04-2019. 
of $v$ relative to $\pi$, where $d\left(v, C_{i}\right)=\min \left\{d(v, x) \mid x \in C_{i}\right\}$ is the shortest distance between $v$ and vertices in $C_{i}$. If every two vertices of $G$ have different coordinates relative to $\pi$, then $c$ is said to be a locating $k$-coloring of $G$. The locating-chromatic number of $G$, denoted by $\chi_{L}(G)$, is the least $k$ such that there exists a locating $k$-coloring of $G$. As shown by Chartrand et al. in [1], if $u$ and $v$ are vertices of $G$ such that $d(u, w)=d(v, w)$ for every $w \in V-\{u, v\}$, then $c(u) \neq c(v)$.

In [4], Behtoei and Anbarloei studied the locating chromatic number of friendship graph $\mathrm{Fr}_{t}$, which is the graph obtained by joining the complete graph $K_{1}$ to the $t$ disjoint copies of $K_{2}$. They showed that $\chi_{L}\left(F r_{t}\right)=1+\min \left\{k \mid t \leq\left(\begin{array}{l}k \\ 2\end{array}\right)\right\}$. In this paper, we study some subdivisions of $F r_{t}$ and their locating-chromatic numbers. In general, a subdivision of a graph $G$ is a graph obtained by replacing some edges of $G$, say $\epsilon_{1}, \epsilon_{2}, \ldots, \epsilon_{r}$, respectively with paths $P_{1}, P_{2}, \ldots, P_{r}$ of length one or greater, where these paths may differ in length. In particular, when we say a subdivision of a graph on some edges $l \geq 0$ times, we are specifying which or how many edges are replaced and ensuring the paths replacing the edges are all of length $l+1$. Purwasih et al. [9] showed that $\chi_{L}(G) \leq 1+\chi_{L}(H)$ if $G$ is a subdivision of a graph $H$ on one edge. We investigate the case where $H=F r_{t}$ by determining the locating-chromatic number of any subdivision of $F r_{t}$ on one edge and also the locating-chromatic number of any subdivision of $F r_{t}$ once on each of its cycle. We also give a tight upper bound for any subdivision of $F r_{t}$. Throughout this paper, for $t \geq 2$ we denote the center of $F r_{t}$, that is the vertex with the largest degree, by $z$. For every natural number $n$, we also denote $[n]=\{1,2, \ldots, n\}$.

\section{MAIN RESULTS}

In this section, we determine the locating-chromatic number of any subdivision of $\mathrm{Fr}_{t}$ on one edge. We also determine the locating-chromatic number of any subdivision of $F r_{t}$ once on each of its cycle.

2.1. Subdivision of $F r_{t}$ on one edge. Throughout this subsection, let $t \geq 2$ and $l \geq 1$ be two natural numbers and let $G$ be a subdivision of $F r_{t}$ on one edge $l$ times. For each $n \geq 3$, we define $d_{n}=\left(\begin{array}{c}n-1 \\ 2\end{array}\right)+1$. Observe that if $t \geq 3$, we have $d_{k-1}<t \leq d_{k}$ for some $k \in\{4,5,6, \ldots\}$. We begin with the following lemmas.

Lemma 1. If $3 \leq t=d_{k}$ and $l=2$, then $\chi_{L}(G)=k+1$.

Proof. By definition of $G$, there are exactly $d_{k}-1$ number of 3-cycles and a 5 -cycle in $G$. Consider the collection of 2 -subsets of $[k-1]$, denoted by $[k-1]^{2}$. Since $\left|[k-1]^{2}\right|=\left(\begin{array}{c}k-1 \\ 2\end{array}\right)$, we can denote the elements of $[k-1]^{2}$ by $u_{1}, u_{2}, \ldots$, and $u_{\left(\begin{array}{c}k-1 \\ 2\end{array}\right)}$.

Now, we start by assigning colors to the vertices of $G$. We immediately assign the color $k+1$ to the vertex $z$. To assign colors to other vertices, observe that since there are $d_{k}-1=\left(\begin{array}{c}k-1 \\ 2\end{array}\right)$ number of 3 -cycles, there are $2\left(\begin{array}{c}k-1 \\ 2\end{array}\right)$ vertices other than $z$ that lie on a 3 -cycle. We denote these vertices by $v_{1}, v_{2}, \ldots$, and $v_{2\left(\begin{array}{c}k-1 \\ 2\end{array}\right)}$, where $v_{2 i-1}$ and $v_{2 i}$ are on the same 3 -cycle for each $i=1,2, \ldots,\left(\begin{array}{c}k-1 \\ 2\end{array}\right)$. If we write 
$u_{i}=\left\{a_{i}, b_{i}\right\}$ where $a_{i}<b_{i}$, assign the color $a_{i}$ and $b_{i}$ respectively to $v_{2 i-1}$ and $v_{2 i}$. To finish the color assignment, let the 5 -cycle in $G$ be $z w_{1} w_{2} w_{3} w_{4} z$. Assign the colors $1, k+1, k$, and 1 respectively to $w_{1}, w_{2}, w_{3}$, and $w_{4}$. Let $c$ be the obtained coloring. Clearly, $c$ is a well-defined graph coloring since no two adjacent vertices are assigned the same color.

We show that $c$ is a locating coloring. Let $x$ and $y$ be two vertices with the same color. If $x$ and $y$ are in the same cycle, then the only possibilities are, without loss of generality, either $(x, y)=\left(z, w_{2}\right)$ or $(x, y)=\left(w_{1}, w_{4}\right)$. However, in both of these cases, the $k$-th component of the coordinate of $x$ and $y$ differ since $2=d\left(x, w_{3}\right) \neq d\left(y, w_{3}\right)=1$ and $w_{3}$ is the only vertex colored $k$.

Let us now assume that $x$ and $y$ are in different cycles. If both are in different 3 -cycles, clearly their coordinates differ since their neighbors other than $z$ have different colors by definition of $u_{1}, u_{2}, \ldots$, and $u_{\left(\begin{array}{c}k-1 \\ 2\end{array}\right)}$. If, without loss of generality, $x$ is in a 5-cycle and $y$ is in a 3 -cycle, then either $x=w_{1}$ or $x=w_{4}$ since the colors $k$ and $k+1$ are not assigned to $y$. In both cases, however, their neighbors other than $z$ also have different colors. Hence, their coordinates differ. Thus, we have shown that $c$ is a locating $(k+1)$-coloring and that $\chi_{L}(G) \leq k+1$.

We now show that $\chi_{L}(G)>k$ by contradiction. Suppose that there exists a locating $k$-coloring $c^{\prime}$ for $G$. Suppose that $c^{\prime}(z)=k$. Hence, without loss of generality, the pair of vertices $\left\{v_{2 i-1}, v_{2 i}\right\}$ has to be assigned by the pair of colors $u_{i}=\left\{a_{i}, b_{i}\right\}$ for each $i=1,2, \ldots,\left(\begin{array}{c}k-1 \\ 2\end{array}\right)$. Moreover, without loss of generality, let $c^{\prime}\left(w_{1}\right)=1$. If $c^{\prime}\left(w_{2}\right) \neq k$, then we let $c^{\prime}\left(w_{2}\right)=m \in\{2,3, \ldots, k-1\}$. However, there are two vertices $v_{p}$ and $v_{p+1}$ such that $\left(c^{\prime}\left(v_{p}\right), c^{\prime}\left(v_{p+1}\right)\right)=(1, m)$. Observe that $d\left(w_{1}, w\right)=d\left(v_{p}, w\right)$ for any vertex $w$ that is assigned by any color other than 1. Hence, $w_{1}$ and $v_{p}$ have the same coordinate, contradicting the definition of a locating coloring. Thus, we must have $c^{\prime}\left(w_{2}\right)=k$. However, by the same argument, we must also have $c^{\prime}\left(w_{3}\right)=k$, which contradicts the definition of coloring. Thus, we have shown that $\chi_{L}(G)>k$ and we conclude that $\chi_{L}(G)=k+1$.

Lemma 2. If $3 \leq t=d_{k}$ and $l \neq 2$, then $\chi_{L}(G)=k$.

Proof. Since $t=d_{k}$ and $l \neq 2$, there are exactly $d_{k}-1$ number of 3 -cycles and an $(l+3)$-cycle in $G$. We start by coloring $G$. Assign the color $k$ to the vertex $z$ and assign colors to the vertices lying in 3-cycles other than $z$ by using the same way used in the proof of the previous lemma. Consider these cases.

a. Suppose that the $(l+3)$-cycle is $z s_{1} s_{2} \ldots s_{4 q-1} \mathrm{z}$ for some $q$. Assign the color $k$ to $s_{2}, s_{4}, \ldots, s_{4 q-2}$. Assign the color 1 to $s_{1}, s_{3}, \ldots, s_{2 q-1}$. Assign the color 2 to $s_{2 q+1}, s_{2 q+3}, \ldots, s_{4 q-1}$. Let $c$ be the resulting coloring. Clearly, $c$ is a well-defined coloring. We show that $c$ is a locating coloring. Let $x$ and $y$ be two vertices with the same color. If $x$ and $y$ are in the same cycle, then both have to be in the $(l+3)$-cycle. If both are colored $k$ and their neighbors are only vertices of color 1 , then their coordinates differ by their distances to a vertex colored 3 since $t=3$, or other colors other than 1 and 2 . It is also the case when their neighbors are only vertices of color 2 . If 
their neighbors are vertices of color 1 and 2, one of them is $z$ and the other one is $s_{2 q}$. In this case, their coordinates also differ by their distances to a vertex colored other than 1 and 2 . The same argument also applies if the color of $x$ and $y$ are 1 or 2 . Moreover, if $x$ is in the $(l+3)$-cycle and $y$ is in a 3-cycle without loss of generality, then $x$ and $y$ are not colored $k$. However, the neighbors of $x$ are only vertices colored $k$, while some of the neighbors of $y$ are not colored $k$. Hence, their coordinates differ. Thus, $c$ is a locating $\mathrm{k}$-coloring.

b. Suppose that the $(l+3)$-cycle is $z s_{1} s_{2} \ldots s_{4 q-3} z$ for some $q$. Assign the color $k$ to $s_{2}, s_{4}, \ldots, s_{4 q-4}$. Assign the color 1 to $s_{1}, s_{3}, \ldots, s_{2 q-1}$. Assign the color 2 to $s_{2 q+1}, s_{2 q+3}, \ldots, s_{4 q-3}$. Let $c$ be the resulting coloring. Clearly, $c$ is a well-defined coloring. We show that $c$ is a locating coloring. Let $x$ and $y$ be two vertices with the same color. By using the same argument as in part a, we see that $c$ is indeed a locating $k$-coloring.

c. Suppose that the $(l+3)$-cycle is a $(2 q-1)$-cycle $z s_{1} s_{2} \ldots s_{2 q-2} z$. Assign the color $k$ to $s_{2}, s_{4}, \ldots, s_{2 q-6}$ and $s_{2 q-3}$. Assign the color 1 to $s_{1}, s_{3}$, $\ldots, s_{2 q-5}$. Assign the color 2 to $s_{2 q-2}$ and $s_{2 q-4}$. Let $c$ be the resulting coloring. Clearly, $c$ is well-defined. The argument to show that $c$ is indeed a locating $k$-coloring is similar to part a or part b with minor difference, that is if $x$ and $y$ are two vertices of color $k$ other than $z$, then their neighbors are either only vertices of color 1 or only vertices of color 2 .

Thus, we have $\chi_{L}(G) \leq k$. We now show that $\chi_{L}(G)>k-1$ by contradiction. Suppose that there exists a $(k-1)$-coloring $c^{\prime}$ for $G$. Let $c^{\prime}(z)=k-1$. Since $z$ is adjacent to all vertices in the 3-cycles, the colors of those vertices have to be in $[k-2]$. However, there are more than $\left(\begin{array}{c}k-2 \\ 2\end{array}\right)$ number of 3 -cycles in $G$, while the cardinality of $[k-2]^{2}$ is $\left(\begin{array}{c}k-2 \\ 2\end{array}\right)$. Hence, by the pigeon-hole principle, there exist two pairs of vertices lying in 3-cycle, say $\left\{a_{1}, b_{1}\right\}$ and $\left\{a_{2}, b_{2}\right\}$, where their elements are different, such that $\left\{c^{\prime}\left(a_{1}\right), c^{\prime}\left(b_{1}\right)\right\}=\left\{c^{\prime}\left(a_{2}\right), c^{\prime}\left(b_{2}\right)\right\}$. Let $a_{1}$ and $b_{1}$ are colored the same as $a_{2}$ and $b_{2}$, respectively. However, the distances of $a_{1}$ and $a_{2}$ to a vertex colored other than $c^{\prime}\left(a_{1}\right)=c^{\prime}\left(a_{2}\right)$ is equal. This means their coordinates are equal, contradicting the definition of locating coloring. Thus, we have $\chi_{L}(G)>k-1$ so that $\chi_{L}(G)=k$.

Lemma 3. If $3 \leq t<d_{k}$, then $\chi_{L}(G)=k$.

Proof. We start by coloring the graph $G$. Assign the color $k$ to $z$. Assign colors to vertices lying in 3-cycles other than $z$ by using the same way used in the proof of the first lemma, that is by taking different elements in the set $[k-1]^{2}$ as pairs of colors for pairs of vertices in each 3-cycle. Since $t<d_{k}$, there are less than $\left(\begin{array}{c}k-1 \\ 2\end{array}\right)$ number of 3-cycles, so that there exist elements in $[k-1]^{2}$ that are not used as a pair of color in any 3-cycle. Denote this element by $\left(g_{1}, g_{2}\right)$.

Suppose that the $(l+3)$-cycle in $G$ is $z s_{1} s_{2} \ldots s_{l+2} z$. If $l+3$ is odd, use the colors $g_{1}, g_{2}, g_{1}, g_{2}, \ldots, g_{1}, g_{2}$ respectively to color $s_{1}, s_{2}, s_{3}, s_{4}, \ldots, s_{l+2}$. Otherwise, assign the color $k$ to the vertex $s_{\frac{l+3}{2}}$ and use the colors $g_{1}, g_{2}, g_{1}, g_{2}, \ldots$ respectively 
to color $s_{1}, s_{2}, s_{3}, s_{4}, \ldots, s_{j}$, where $j=\frac{l+3}{2}-1$, and use the colors $g_{2}, g_{1}, g_{2}, g_{1}, \ldots$ respectively to color $s_{l+2}, s_{l+1}, s_{l}, s_{l-1}, \ldots, s_{j+2}$.

It is easy to see, by using the same argument as in the proof of previous lemma, that all vertices colored the same have different coordinates. In this case, vertices colored $g_{1}$ and $g_{2}$ create the differences. Hence, $\chi_{L}(G) \leq k$. The proof showing that $\chi_{L}(G)>k-1$ is similar to the last paragraph of the proof of the last lemma. Thus, we have $\chi_{L}(G)=k$.

From previous three lemmas, we have the following theorem.

Theorem 1. Let $t \geq 3$ and $l \geq 1$ be two natural numbers. Let $G$ be a subdivision of $F r_{t}$ on one edge $l$ times. For each $n \geq 3$, let $d_{n}=\left(\begin{array}{c}n-1 \\ 2\end{array}\right)+1$ and $d_{k-1}<t \leq d_{k}$ for some $k$. Hence, we have $\chi_{L}(G)=k+1$ if $t=d_{k}$ and $l=2$, and $\chi_{L}(G)=k$ otherwise.

We treat the case $t=2$ separately in the next proposition.

Proposition 1. Let $l \geq 1$ be a natural number. If $G$ is a subdivision of $F r_{2}$ on one edge $l$ times, then $\chi_{L}(G)=4$.

Proof. We start by coloring $G$. Assign the color 4 to the vertex $z$. Assign the color 1 and 2 to the two vertices lying in the only existing 3-cycle. Now, denote the $(l+3)$-cycle in $G$ by $z u_{1} u_{2} \ldots u_{l+2} z$.

Assume first that $l$ is even. Assign the colors $1,3,1,3, \ldots, 1,3$ respectively to the vertices $u_{1}, u_{2}, u_{3}, u_{4}, \ldots, u_{l+2}$. By doing this, the vertices colored 3 have their coordinates differed by their distances to the vertex colored 4 , and the vertices colored 1 have their coordinates differed by their distances to the vertex colored 2 . Thus, we obtain a locating 4-coloring.

Assume now that $l$ is odd. Assign the color 4 to the vertex $u_{\frac{l+3}{2}}$. Assign the color 1 to each vertex of the form $u_{1}, u_{3}, \ldots, u_{l_{1}}$, where $l_{1}<\frac{l+3}{2}$, and vertex of the form $u_{l_{2}}, \ldots, u_{l-1}, u_{l+1}$, where $l_{2}>\frac{l+3}{2}$. Assign the color 3 to other remaining vertices. By doing this, the vertices colored 3 have their coordinates differ by their distances to the vertex colored 2 , and so do the vertices colored 1 . Thus, we obtain a locating 4-coloring.

We have shown that $\chi_{L}(G) \leq 4$. We now show that $\chi_{L}(G)>3$. Suppose that there exists a locating 3 -coloring on $G$. Without loss of generality, assume that the 3 -cycle in $G$ is colored by 1,2 , and 3 , where $z$ is assigned the color 3 . Suppose that there exists a vertex colored by 2 in the $(l+3)$-cycle in $G$. Let $j$ be the least index such that $u_{j}$ is colored by 2 . If $j$ is odd, then the vertices $u_{1}, u_{3}, \ldots, u_{j-2}$ have to be colored by 1 since the color of $z$ is 3 , and we must also have the vertices $u_{2}, u_{4}, \ldots, u_{j-1}$ colored by 3 . However, the coordinate of $u_{j-1}$ is equal to the coordinate of $z$, contradicting the definition of locating coloring. If $j$ is even instead, then the vertices $u_{1}, u_{3}, \ldots, u_{j-1}$ have to be colored 1 since the color of $z$ is 3 , and we must also have the vertices $u_{2}, u_{4}, \ldots, u_{j-2}$ colored by 3 . 
However, the coordinate of $u_{j-1}$ is equal to the coordinate of the vertex colored by 1 on the 3 -cycle, contradicting again the definition of locating coloring. Hence, there must not be any vertex colored by 2 on the $(l+3)$-cycle. This means that, since $z$ is colored by $3, u_{1}, u_{3}, \ldots, u_{l+2}$ have to be colored by 1 and $u_{2}, u_{4}, \ldots, u_{l+1}$ have to be colored by 3 . However, the vertices $u_{1}$ and $u_{l+2}$ have the same color and the same coordinate, contradicting the definition of the locating coloring. Thus, we have $\chi_{L}(G)=4$.

2.2. Subdivision of $F r_{t}$ once on one edge of each cycle. We now determine the locating-chromatic number of the subdivision of $F r_{t}$ once on one edge of each cycle. This means that each cycle of the graph is a 4 -cycle. Let $G$ be such graph, where $t \geq 2$.

Theorem 2. For each $n \geq 3$, let $e_{n}=\left\lfloor\frac{n-1}{2}\right\rfloor+(n-1)\left\lfloor\frac{n-2}{2}\right\rfloor$ and $e_{k-1}<t \leq e_{k}$ for some $k$. Hence, we have $\chi_{L}(G)=k$.

Proof. We define a locating $\chi_{L}(G)$-coloring $c: V \rightarrow[k]$ on $G$. We first set $c(z):=k$. Assume that $C(1), C(2), \ldots, C(t)$ are all of the 4-cycles in $G$ and denote $C(i)$ by $z u_{i, 1} u_{i, 2} u_{i, 3} z$ for each $i$. Clearly, $k \geq 4$ since $2 \leq t \leq e_{k}$.

Define a 3 -tuple $W_{i}^{\prime}:=\left(w_{i, 1}^{\prime}, w_{i, 2}^{\prime}, w_{i, 3}^{\prime}\right)$ with $w_{i, 1}^{\prime}:=2 i-1, w_{i, 2}^{\prime}:=k, w_{i, 3}^{\prime}:=2 i$ for $i=1,2, \ldots,\left\lfloor\frac{k-1}{2}\right\rfloor$. Next, for $j=1,2, \ldots,(n-1)\left\lfloor\frac{k-2}{2}\right\rfloor$, define a 3 -tuple $W_{j}^{\prime \prime}:=\left(w_{i, 1}^{\prime \prime}, w_{i, 2}^{\prime \prime}, w_{i, 3}^{\prime \prime}\right)$ with

$$
\begin{aligned}
& \left(w_{i, 1}^{\prime \prime}, w_{i+1,1}^{\prime \prime}, \ldots, w_{i+\left\lfloor\frac{k-2}{2}\right\rfloor-1,1}^{\prime \prime}\right):=\left(i+1, i+3, \ldots, i+2\left\lfloor\frac{k-2}{2}\right\rfloor-1\right), \\
& \left(w_{i, 2}^{\prime \prime}, w_{i+1,2}^{\prime \prime}, \ldots, w_{i+\left\lfloor\frac{k-2}{2}\right\rfloor-1,2}^{\prime \prime}\right):=(i, i, \ldots, i), \\
& \left(w_{i, 3}^{\prime \prime}, w_{i+1,3}^{\prime \prime}, \ldots, w_{i+\left\lfloor\frac{k-2}{2}\right\rfloor-1,3}^{\prime \prime}\right):=\left(i+2, i+4, \ldots, i+2\left\lfloor\frac{k-2}{2}\right\rfloor\right)
\end{aligned}
$$

for $i=1,2, \ldots, k-1$, by noting that the components are calculated under modulo $k-1$. Observe that in $W_{j}^{\prime \prime}$, there is no entry that is equal to $k$. Observe also that $W_{i}^{\prime}$ and $W_{j}^{\prime \prime}$ never equal to each other since their second entries differ for any $i$ and $j$. By definition, we also see that $W_{i_{1}}^{\prime}$ and $W_{i_{2}}^{\prime}$ differ for any different $i_{1}$ and $i_{2}$, and that $W_{j_{1}}^{\prime \prime}$ and $W_{j_{2}}^{\prime \prime}$ differ for any different $j_{1}$ and $j_{2}$. Hence, if we write $W:=\left\{W_{i}^{\prime} \mid i=1,2, \ldots,\left\lfloor\frac{k-1}{2}\right\rfloor\right\} \cup\left\{W_{j}^{\prime \prime} \mid j=1,2, \ldots,(k-1)\left\lfloor\frac{k-2}{2}\right\rfloor\right\}$, we have $|W|=e_{k}$. We can then write $W=\left\{W_{1}, W_{2}, \ldots, W_{e_{k}}\right\}$. Now, for each $i \in[t]$, define the coloring $c[C(i)]:=\left(c\left(u_{i, 1}\right), c\left(u_{i, 2}\right), c\left(u_{i, 3}\right)\right):=W_{i}$.

From the definition of $W$, clearly $c$ is a $k$-coloring. We now show that $c$ is a locating coloring. Let $x$ and $y$ be two different vertices with the same color in $G$. If $x=z$, then $y=u_{i, 2}$ for some $i$ such that $c\left(u_{i, 2}\right)=k$, if it exists. However, since $t \geq 2$ and by definition of $c$, the vertex $x$ is adjacent to vertices with colors other than $c\left(u_{i, 1}\right)$ and $c\left(u_{i, 3}\right)$, while $y$ is only adjacent to vertices with these colors. Hence, the coordinates of $x$ and $y$ differ, so we assume that $x$ and $y$ are not $z$.

Now, let our $x$ and $y$ be in the 4 -cycles $C\left(i_{1}\right)$ and $C\left(i_{2}\right)$, respectively, where $i_{1}$ and $i_{2}$ are two different elements of $[t]$. 
Let $c\left[C\left(i_{1}\right)\right]$ and $c\left[C\left(i_{2}\right)\right]$ both be in $\left\{W_{i}^{\prime} \mid i=1,2, \ldots,\left\lfloor\frac{k-1}{2}\right\rfloor\right\}$. Hence, we must have $x=u_{i_{1}, 2}$ and $y=u_{i_{2}, 2}$, or vice-versa. However, the colors of the neighbors of $x$ clearly differ from the colors of the neighbors of $y$. Thus, their coordinates differ.

Let $c\left[C\left(i_{1}\right)\right]$ and $c\left[C\left(i_{2}\right)\right]$ both be in $\left\{W_{j}^{\prime \prime} \mid j=1,2, \ldots,(k-1)\left\lfloor\frac{k-2}{2}\right\rfloor\right\}$. There are some cases to consider. For the first case, if $x=u_{i_{1}, 2}$ and $y=u_{i_{2}, 2}$, then clearly they have different coordinates by looking at the colors of their neighbors. For the next case, if $x=u_{i_{1}, 2}$ and $y=u_{i_{2}, 1}$ (or $y=u_{i_{2}, 3}$ without loss of generality), then $y$ is adjacent to $z$, which is a vertex colored $k$, but $x$ is not adjacent to any vertex colored $k$, so we know that their coordinates differ. For the last case, if $x=u_{i_{1}, 1}$ and $y=u_{i_{2}, 1}$ (without loss of generality), then, by definition of $c$, the colors of $u_{i_{1}, 2}$ and $u_{i_{2}, 2}$ differ, so that the colors of the neighbors of $x$ and $y$ also differ, and hence $x$ and $y$ have different coordinates.

Let $c\left[C\left(i_{1}\right)\right] \in\left\{W_{j}^{\prime \prime} \mid j=1,2, \ldots,(k-1)\left\lfloor\frac{k-2}{2}\right\rfloor\right\}$ and $c\left[C\left(i_{2}\right)\right] \in\left\{W_{j}^{\prime \prime} \mid j=\right.$ $\left.1,2, \ldots,(k-1)\left\lfloor\frac{k-2}{2}\right\rfloor\right\}$ (without loss of generality). Thus, we have, say $x=u_{i_{1}, 1}$, and $y=u_{i_{2}, 2}$ or $y=u_{i_{2}, 1}$. However, both neighbors of $x$ are colored $k$, but only one of the neighbors of $k$ is colored $k$. Hence, their coordinates differ.

Thus, we have shown that $c$ is a locating $k$-coloring, so that $\chi_{L}(G) \leq k$.

We now show that $\chi_{L}(G)>k-1$. Suppose that there exists a locating $(k-1)$ coloring on $G$, which we denote by $c^{\prime}$. Assume that $c^{\prime}(z)=k-1$. We divide all of the 4 -cycles into $k-1$ types. Type $a$ consists of all 4 -cycles $C(i)=z u_{i, 1} u_{i, 2} u_{i, 3} z$ with $c^{\prime}\left(u_{i, 2}\right)=a$. Observe that if there exist two 4-cycles of type $k-1$, say $C(i)$ and $C(j)$, where $c^{\prime}\left(u_{i, 1}\right)=c^{\prime}\left(u_{j, 1}\right)$ without loss of generality, then the coordinates of $u_{i, 1}$ and $u_{j, 1}$ must be the same since both are adjacent only to two vertices colored $k-1$ and $d\left(u_{i, 1}, u_{i, 3}\right)=d\left(u_{i, 1}, z\right)+d\left(z, u_{i, 3}\right)=d\left(u_{j, 1}, z\right)+d\left(u_{j, 1}, u_{i, 3}\right)=2$. Moreover, $2=d\left(u_{j, 1}, u_{j, 3}\right)=d\left(u_{i, 1}, u_{j, 3}\right)$ and $d\left(u_{i, 1}, x\right)=d\left(u_{i, 1}, z\right)+d(z, x)=d\left(u_{j, 1}, z\right)+$ $d(z, x)=d\left(u_{j, 1}, x\right)$ for each vertex $x$ that is not $u_{i, 1}, u_{i, 2}, u_{i, 3}, u_{j, 1}, u_{j, 2}, u_{j, 3}$. Hence, since $u_{i, 1}$ and $u_{j, 1}$ must not be colored $k-1$, there are at most $\left\lfloor\frac{k-2}{2}\right\rfloor$ number of 4-cycles of type $k-1$ by the pigeon-hole principle.

Let $C(i)$ be a 4 -cycle of type $b$ where $c^{\prime}\left(u_{i, 2}\right)=b \in[k-2]$. By the similar observation to the previous paragraph, there are at most $\left\lfloor\frac{k-3}{2}\right\rfloor$ number of 4-cycles of type $b$. Hence, there are at most $(k-2)\left\lfloor\frac{k-3}{2}\right\rfloor$ number of 4 -cycles of type other than $k-1$. Thus, by combining with the previous paragraph, there are at most $e_{k-1}$ number of 4-cycles in $G$. This contradicts the assumption on $t$. We conclude that $\chi_{L}(G)>k-1$, so that $\chi_{L}(G)=k$.

2.3. Upper bound for arbitrary subdivision of $F r_{t}$. We now study the upper bound for arbitrary subdivision of $F r_{t}$. It is known that $\chi_{L}(G) \leq 1+\chi_{L}(H)$ if $G$ is a subdivision of a graph $H$ on one edge. For $H=F r_{t}$, this bound is strengthened.

Theorem 3. If $G$ is a subdivision of $F r_{t}$ where $t \geq 2$, then we have $\chi_{L}(G) \leq$ $\chi_{L}\left(F r_{t}\right)$. Precisely, if $\left(\begin{array}{c}k-2 \\ 2\end{array}\right)<t \leq\left(\begin{array}{c}k-1 \\ 2\end{array}\right)$, we have $\chi_{L}(G) \leq k$. 
Proof. Clearly, $k \geq 4$. We construct a locating $k$-coloring $c: V(G) \rightarrow[k]$ on $G$. We start by setting $c(z):=k$. Let $C(1), C(2), \ldots, C(t)$ denote all the cycles in $G$. We start with the first case where $t=\left(\begin{array}{c}k-1 \\ 2\end{array}\right)$.

Suppose that there is no 4-cycle in $G$. Write $C(i)=w_{i, 1} w_{i, 2} \ldots w_{i, s(i)} w_{i, 1}$ where $s(i) \neq 4$ and $w_{i, 1}=z$ for each $i$. Assume that $[k-1]^{2}=\left\{u_{1}, u_{2}, \ldots, u_{t}\right\}$. If $s(i)$ is odd, then set $c\left(w_{i, 2}\right):=c\left(w_{i, 4}\right):=\ldots:=c\left(w_{i, s(i)-1}\right):=a_{i}$ and $c\left(w_{i, 3}\right):=$ $c\left(w_{i, 5}\right):=\ldots:=c\left(w_{i, s(i)}\right):=b_{i}$, where $\left\{a_{i}, b_{i}\right\}:=u_{i}$. If $s(i)$ is even, then set $c\left(w_{i, 2}\right):=c\left(w_{i, 4}\right):=\ldots:=c\left(w_{i, s_{1}(i)}\right):=a_{i}, c\left(w_{i, s(i)-1}\right):=c\left(w_{i, s(i)-3}\right):=$ $\ldots:=c\left(w_{i, s_{2}(i)}\right):=a_{i}, c\left(w_{i, 3}\right):=c\left(w_{i, 5}\right):=\ldots:=c\left(w_{i, s_{3}(i)}\right):=b_{i}, c\left(w_{i, s(i)}\right):=$ $c\left(w_{i, s(i)-2}\right):=\ldots:=c\left(w_{i, s_{4}(i)}\right):=b_{i}$, and $c\left(w_{i, \frac{s(i)}{2}+1}\right):=k$, where $\left\{a_{i}, b_{i}\right\}:=$ $u_{i}, s_{1}(i)<\frac{s(i)}{2}+1, s_{2}(i)>\frac{s(i)}{2}+1, s_{3}(i)<\frac{s(i)}{2}+1$, and $s_{4}(i)>\frac{s(i)}{2}+1$. Observe that two adjacent vertices in $G$ lie in a $C(i)$. By definition of $c$, those two vertices have different colors. Thus, $c$ is a $k$-coloring.

Next, to show that $c$ is locating coloring, let $z_{1}$ and $z_{2}$ be two different vertices having the same color in $G$, that is $c\left(z_{1}\right)=c\left(z_{2}\right)=a_{0}$. If one of $z_{1}$ or $z_{2}$ is $z$, say $z_{1}$, then we know that, by definition of $c$ and the fact that $t \geq 2, z_{1}$ is adjacent to at least 4 vertices which are two vertices in the cycle where $z_{2}$ belongs and two other vertices in another cycle, and that three of these four vertices have different colors. However, $z_{2}$ is only adjacent to at most two vertices with different colors. Hence, the coordinates of $z_{1}$ and $z_{2}$ differ. Now, let $z_{1}$ and $z_{2}$ be vertices other than $z$. Assume that both are in different cycles, say $C\left(i_{1}\right)$ and $C\left(i_{2}\right)$, respectively. If $a_{0}=k$, then $C\left(i_{1}\right)$ and $C\left(i_{2}\right)$ are cycles of even length by definition of $c$. Again, by definition of $c, z_{1}$ and $z_{2}$ are vertices that have their distances to $z$ the greatest in the cycles containing them, so that $z_{1}$ is adjacent to two vertices colored $a_{i_{1}}$ and $b_{i_{1}}$, and $z_{2}$ is adjacent to two vertices colored $a_{i_{2}}$ and $b_{i_{2}}$, but $u_{i_{1}} \neq u_{i_{2}}$. Thus, the coordinates of $z_{1}$ and $z_{2}$ are different. If $a_{0} \neq k$, then, since no cycle is a 4 -cycle and each of $z_{1}$ and $z_{2}$ has a neighbor $z_{1}^{\prime}$ and $z_{2}^{\prime}$, respectively, that $c\left(z_{1}^{\prime}\right) \neq c\left(z_{2}^{\prime}\right)$ by definition, the coordinates of $z_{1}$ and $z_{2}$ are different.

Now, let $z_{1}$ and $z_{2}$ be in the same cycle $C(i)$, and both are not $z$. By definition of $c$, we have $a_{0} \neq k$. Again by definition of $c$ and the fact that $t \geq 2$, there exists a vertex $z^{\prime}$ colored $a_{0}^{\prime}$ outside of $C(i)$ and no vertex in $C(i)$ is colored $a_{0}^{\prime}$. By the numbering of $C(i)$, we have $d\left(z_{1}, z^{\prime}\right)=d\left(z_{1}, z\right)+d\left(z, z^{\prime}\right) \neq d\left(z_{2}, z\right)+d\left(z, z^{\prime}\right)=$ $d\left(z_{2}, z^{\prime}\right)$. Hence, the coordinates of $z_{1}$ and $z_{2}$ differ. Thus, we have shown that $c$ is a locating coloring and that $\chi_{L}(G) \leq k$.

For the next case, suppose that there are $q$ number of 4-cycles in $G$. We show that $\chi_{L}(G) \leq k$. Write $q=(k-1) m+r$ where $r$ and $m$ are unique integers satisfying $0 \leq r<k-1$ and $m \geq 0$ by the division algorithm. Let the 4 -cycles be denoted by $Q_{1}, Q_{2}, \ldots, Q_{q}$. Consider the complete graph $H$ on the set $[k-1]$.

Assume that $k$ is odd. We must have $m \leq \frac{k-1}{2}-1$, otherwise we would have $q>\left(\begin{array}{c}k-1 \\ 2\end{array}\right)$, which is a contradiction. Since $k-1$ is even, by decomposing $\mathrm{H}$ to obtain its Hamiltonian cycles and its 1-factors, there exist subgraphs $H_{1}, H_{2}, \ldots, H_{\frac{k-1}{2}-1}, E_{1}, E_{2}, \ldots, E_{\frac{k-1}{2}}$ of $H$. We continue by noting that $H_{i}$ is 
a Hamiltonian cycle and $H_{i}$ and $H_{j}$ are edge-disjoint subgraphs for each different $i$ and $j$, and that $E_{i}$ is a complete graph on two vertices and $E_{i}$ and $E_{j}$ are edge-disjoint subgraphs for each different $i$ and $j$.

For each $p \in[m]$, consider the $k-1$ number of 4 -cycles $Q_{(k-1)(p-1)+1}$, $Q_{(k-1)(p-1)+2}, \ldots, Q_{(k-1) p}$. We define the coloring $c$ on these cycles that is associated with the subgraph $H_{p}$. Let us write $H_{p}=h_{p, 1}, h_{p, 2}, \ldots, h_{p, k-1}, h_{p, 1}$. There exist three vertices $v_{p, j, 1}, v_{p, j, 2}, v_{p, j, 3}$ that are not $z$ on $Q_{(k-1)(p-1)+j}$ where $j \in[k-1]$. Set $c\left(v_{p, j, 1}\right):=h_{p, j}, c\left(v_{p, j, 2}\right):=h_{p, j+1}, c\left(v_{p, j, 3}\right):=h_{p, j+2}$, where $j, j+1$, and $j+2$ are calculated under modulo $k-1$. By this definition, adjacent vertices on these cycles have different colors.

For the case $r>\frac{k-1}{2}+1$, we have $m<\frac{k-1}{2}-1$, so that there exist a Hamiltonian cycle $H_{m+1}$ that have not yet been associated with the 4-cycles on the previous paragraph. We define the coloring $c$ on the 4-cycles $Q_{(k-1) m+1}, Q_{(k-1) m+2}, \ldots$, $Q_{(k-1) m+r}$ associated with the subgraph $H_{m+1}$. Similar to the previous paragraph, by changing the role of $p$ with $m+1$ and $j \in[r]$, and when $j=r$, we set $c\left(v_{m+1, j, 2}\right):=k$, we see that adjacent vertices on these cycles have different colors.

For the case $r \leq \frac{k-1}{2}$, consider the $r$ number of 4-cycles $Q_{(k-1) m+1}, Q_{(k-1) m+2}$, $\ldots, Q_{(k-1) m+r}$. We define a coloring $c$ on the cycle $Q_{(k-1) m+p}$ for each $p \in[r]$ associated with the subgraph $E_{p}$. Assume that $E_{p}=e_{p, 1} e_{p, 2}$. There are three vertices $x_{p, 1}, x_{p, 2}, x_{p, 3}$ that are not $z$ on $Q_{(k-1) m+p}$. Set $c\left(x_{p, 1}\right):=e_{p, 1}, c\left(x_{p, 2}\right):=$ $k, c\left(x_{p, 3}\right):=e_{p, 2}$. Hence, adjacent vertices on these cycles have different colors.

Note that for the case that $k$ is even, we obtain Hamilton cycles $H_{1}, H_{2}, \ldots$, $H_{\frac{k-2}{2}}$ of $H$. Again, this time we must have $m \leq \frac{k-2}{2}$. The coloring is done by using the similar way to the case that $k$ is odd, except that the case $r>\frac{k-1}{2}+1$ is replaced with the case $r \neq 0$, and the case $r \leq \frac{k-1}{2}$ is not needed. Next, color the remaining $t-q$ cycles by using the similar coloring used to color cycles before there was any 4-cycle, by noting that the pair $\left\{a_{i}, b_{i}\right\}$ that is used is the label of two adjacent vertices that have not been used to color the 4-cycles on the above decomposition. Observe that there are exactly $t-q$ pairs of such labels. Thus, we have shown that $c$ is a $k$-coloring.

We show that $c$ is indeed a locating coloring. Let $x$ and $y$ be two different vertices in $G$ with $c(x)=c(y)$. The cases for $x$ and $y$ that must be considered are:

(1) One of them is $z$

(2) Both are not $z$ and are in the same 4-cycle

(3) Both are not $z$ and are in the same cycle that is not a 4-cycle

(4) Both are not $z, x$ is in a 4-cycle, and $y$ is in a cycle that is not a 4-cycle

(5) Both are not $z$ and are in different 4-cycles

(6) Both are not $z$ and are in different cycles, but these cycles are not 4-cycles.

The first four cases are easily verified. For the fifth case, if both $x$ and $y$ are colored $k$, then, since both are not $z$, their neighbors have to be only two vertices that have the color pair from some labels $E_{i}$ and $E_{j}$, respectively, that are edgedisjoint subgraphs. Hence, the colors of the neighbors of $x$ and $y$ differ. If both are not colored $k$, then the colors of the neighbors of $x$ and $y$ also differ since each two 
4-cycles have their vertices colored based on the labels of the subgraphs of $H$ that are edge-disjoint subgraphs and since $\mathrm{x}$ and $\mathrm{y}$ are different vertices. In fact, if the colors of the neighbors of $x$ and $y$ are only $k$, then, since they belong to different 4-cycles, the colors in both of these cycles must be based on $E_{i}$ and $E_{j}$ that are edge-disjoint subgraphs. This is impossible if $x$ and $y$ are different vertices.

For the sixth case, the same argument also applies by observing the possibilities of the position of $x$ and $y$ and the labels used. Thus, we have shown that $c$ is a locating $k$-coloring so that $\chi_{L}(G) \leq k$.

Lastly, for the case $t<\left(\begin{array}{c}k-1 \\ 2\end{array}\right)$, the $(t+1)$-th, $(t+2)$-th, ..., and so on that have been colored from the coloring on the case $t=\left(\begin{array}{c}k-1 \\ 2\end{array}\right)$ before is removed so that there are $t<\left(\begin{array}{c}k-1 \\ 2\end{array}\right)$ cycles remaining, and the above cases can be verified again the similar way. Thus, the theorem is proved.

\section{REFERENCES}

[1] Chartrand, G., Erwin, D., Henning, M. A., Slater, P. J., and Zhang, P., The locatingchromatic number of a graph, Bull. Inst. Combin. Appl., 36 (2002), 89-101.

[2] Asmiati, Yana, I. K. S. G., and Yulianti, L., On the locating chromatic number of certain barbell graphs, Int. J. Math. Math. Sci., 2018 (2018), retrieved from https://doi.org/10.1155/2018/5327504.

[3] Purwasih, I. A., Baskoro, E. T., Assiyatun, H., Suprijanto, D., and Baca, M., The locatingchromatic number for Halin graphs, Commun. Comb. Optim., 2(1) (2017), 1-9.

[4] Behtoei, A. and Anbarloei, M., The locating chromatic number of the join of graphs, Bull. Iranian Math. Soc., 40(6) (2014), 1491-1504.

[5] Behtoei, A. and Omoomi, B., On the locating chromatic number of the Cartesian product of graphs, Ars Combin., 126 (2016), 221-235.

[6] Syofyan, D. K., Baskoro, E. T., and Assiyatun, H., Trees with certain locating-chromatic number, J. Math. Fund. Sci., 48(1) (2016), 39-47.

[7] Baskoro, E. T. and Asmiati, Characterizing all trees with locating-chromatic number 3, Electronic Journal of Graph Theory and Applications, 1(2) (2013), 109-117.

[8] Behtoei, A. and Anbarloei, M., A bound for the locating chromatic number of trees, Transactions on Combinatorics, 4(1) (2015), 31-41.

[9] Purwasih, I. A., Baskoro, E. T., Assiyatun, H., and Suprijanto, D., The bounds on the locating-chromatic number for a subdivision of a graph on one edge, Procedia Computer Science, 74 (2015), 84-88. 